\title{
Large-scale numerical simulations: Convection in an annular channel rotating about a vertical axis with side-walls
}

\author{
Yingli Chang ${ }^{1}$ and $\mathrm{Yu} \mathrm{Liu}{ }^{2,3}$ \\ ${ }^{1}$ Shanghai fisheries university.334 Jungong Road, Shanghai 200090, China \\ ${ }^{2}$ Shanghai Astronomical Observatory, 80 Nandan Road, Shanghai, China \\ ${ }^{3}$ Graduate University of Chinese Academy of Science, China \\ email: yuliu@shao.ac.cn
}

\begin{abstract}
For the purpose of understanding the dynamics of planetary atmospheres, we use the annular convection model to simulate the dynamics of atmospheres of Jupiter and Saturn. The model (annular channel) rotates about a vertical axis with side-walls, and it is heated from below.

We use the software NaSt3DGP (a parallel software package to solve the 3D incompressible fluid dynamic problems in Cartesian coordinates by using Finite Difference Method) for the computation. It's reliability is tested by our application to simulate fully three-dimensional nonlinear convection in a box with lateral stress-free side-walls, uniformly heated from below. We found that, at moderately large Rayleigh numbers, the complex formation of multiple-jet flows can be maintained by the traveling convective eddies; we also found that the type of the sidewall velocity condition does not play an essential role in determining the primary properties of strongly nonlinear convection.
\end{abstract}

Keywords. Solver, rotation, annular channel, simulation convection

\section{Introduction}

Convection in a rotating Bénard layer has been also studied extensively in attempting to offer valuable insight into the general physics and dynamics in rotating convective systems because of its mathematical simplicity. It is also worth mentioning that the annular system with the gravity vector perpendicular to the axis of rotation, mimicking the equatorial region of a rotating spherical shell, has been extensively studied (for example, Busse, 1970)

Xhinhao Liao et al investigated linear convection and weakly nonlinear differential rotation in an annular channel with the no-slip side-walls, stress-free top and bottom, uniformly heated from below and rotating about a vertical axis, the exactly same model as that studied by Davies-Jones and Gilman (1971).

Our main work will report numerical simulations of nonlinear convection in a box with lateral stress-free side-walls,no-slip and stress-free top and bottom,uniformly heated from below.

\section{Convection in a wide-gap channel}

We first look at the results of numerical simulations of nonlinear convection in a widegap annular channel with stress-free sidewalls. Then we look at the results of threedimensional numerical simulations of no-slip side walls. 

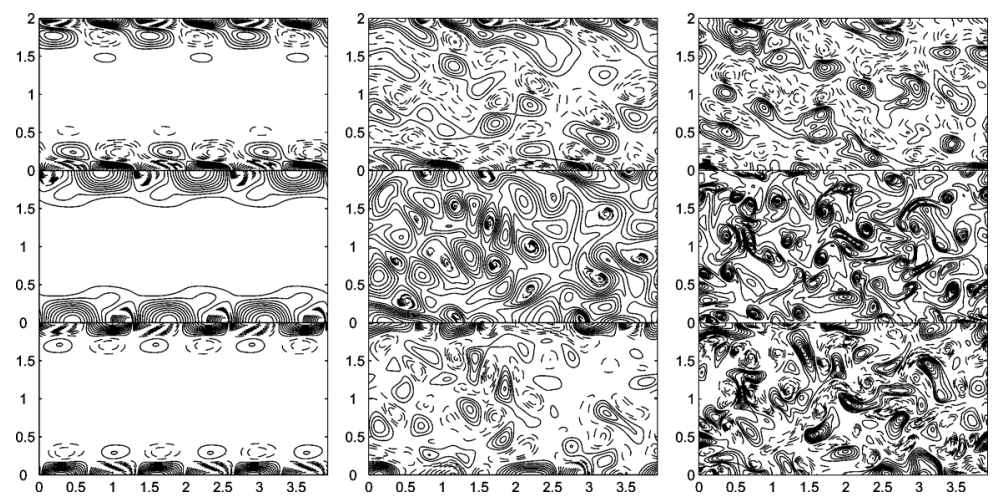

Figure 1. Contours of $u_{x}$ (top), $\Theta$ (middle) and $u_{z}$ (bottom) are shown in a horizontal $x y$-plane. $R=7 \times 10^{4}$ (left), $R=2.5 \times 10^{5}$ (middle), $R=2.5 \times 10^{6}$ (right), $\tau=10^{3}, \Gamma=2, P_{r}=7.0$, stress-free b.c.
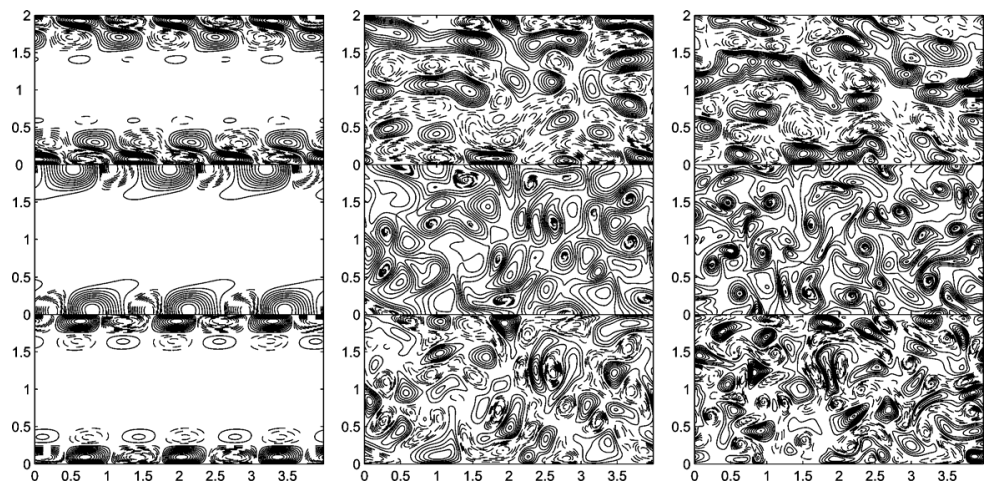

Figure 2. Contours of $u_{x}$ (top), $\Theta$ (middle) and $u_{z}$ (bottom) are shown in a horizontal $x y$-plane. $R=5 \times 10^{4}$ (left), $R=2.5 \times 10^{5}$ (middle), $R=1 \times 10^{6}$ (right), $\tau=10^{3}, P_{r}=7.0$, non-slip side walls.

\section{Discussion}

A major conclusion drawn from the results of the numerical simulations is that at moderately large Rayleigh numbers, the complex formation of multiple-jet flows can be maintained by the travelling convective eddies; and the type of the sidewall velocity condition does not play an essential role in determining the primary properties of strongly nonlinear convection. This is significant with regard to possible geophysical and astrophysical applications because the well-known tangent cylindrical surface in rapidly rotating spherical shells may be regarded as an effective stress-free sidewall.

\section{Acknowledgements}

This work is supported by Ocean Common Weal Cause Research Project in 2008 (2008 05016), Shanghai fund for outstanding youth (10050 810106 4101) and Shanghai fisheries university fund for PHD (A 240106 0342).

\section{References}

Busse, F. H. 1970, J. Fluid Mech. 10, 441-460

Liao, X., Zhang, K., \& Chang, Y. 2005, Geophys. Astrophys. Fluid Dyn. 99, 441-460

Davies-Jones, R. P. \& Gilman P. A. 1971, J. Fluid Mech. 46, 65-81 